\title{
Design of home intelligent robot of internet of things
}

\author{
Guangyu Liang, Yanyang Liu, Kangwei Feng*, Yuefeng Pan, Yaxiong Liu, and Man Yuan \\ College of Automation and Electrical Engineering, Tianjin Vocational And Technical Normal \\ University, Tianjin 300222, China
}

\begin{abstract}
An aging society is the challenge we will face and deal with. It is necessary and important to explore the problems and solutions faced by an aging society. In response to this phenomenon, we designed an IoT home smart robot based on ESP8266 and STM32 to improve the quality of life of the elderly. The robot can realize functions such as environmental monitoring, fire warning, electrical power distribution, light control, auxiliary weight lifting, weather forecast, etc. Connecting to the Baidu cloud platform can realize data visualization, allowing children to remotely view the family's data and equipment conditions in the cloud, and has the function of automatically mopping the floor to reduce the burden of housework. On the basis of the mechanical structure design, combined with the current emerging Internet of Things technology and electronic control technology, mechatronics, the research and development cost is low, the function is complete, and it has strong practicability and innovation.
\end{abstract}

\section{Introduction}

With the aging of the population and the improvement of the quality of life, the Internet of Things home intelligent robots gradually enter the market, and their scale will exceed the industrial robots, and they will be widely used in aging groups and helping the elderly and the disabled. The Internet of Things home intelligent robot can greatly improve the life of the elderly and bring great convenience to the elderly. The research and application of the home intelligent robot of the Internet of Things conforms to the current development direction of intelligent, informatized and networked home life, and has a good research and development prospect.

\section{Overall system design}

The Internet of Things home smart robot uses the STM32 controller as the central processor to monitor the signals from the temperature and humidity sensors, smoke sensors, flame sensors and light intensity sensors in real time. The robot connects to the Baidu cloud

\footnotetext{
* Corresponding author: $\underline{435677646 @ \text { qq.com }}$
} 
platform to realize data visualization, and remotely view the household data and equipment status on the APP. The overall system architecture is shown in Figure 1.

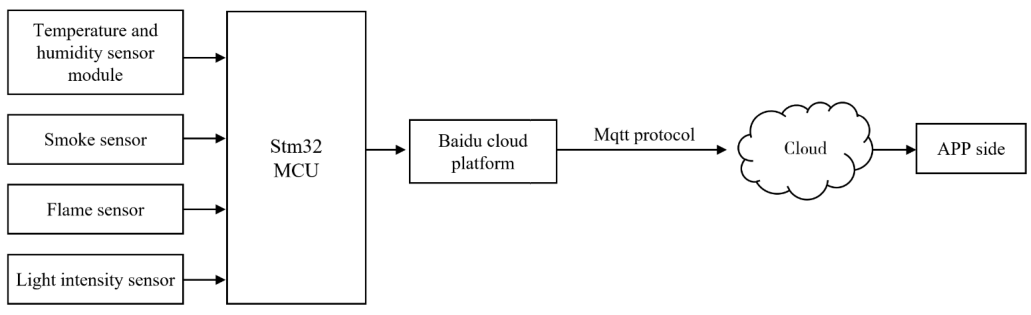

Fig. 1. System overall architecture design.

\section{Mechanical design}

Acrylic board is used as the body, and the mechanical structure is modeled by SolidWorks software. The overall design is divided into three parts, storage structure, lifting structure, and mopping structure.

Using 3D printing technology to print each part of the robot, the model diagram is shown in Figure 2.

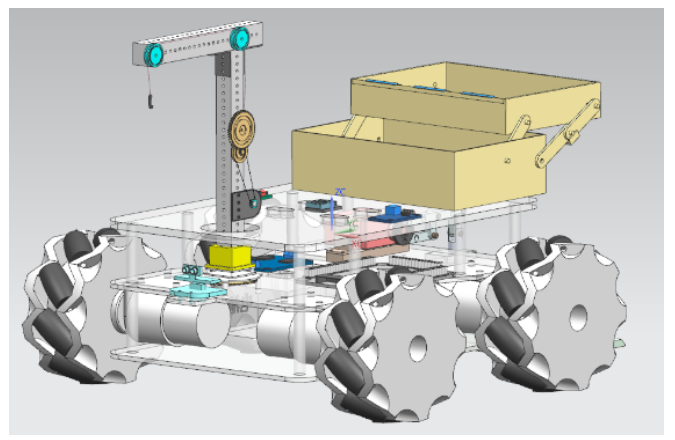

Fig. 2. Mechanical design.

\section{Hardware design}

The STM32 single-chip microcomputer controls the start and stop of each motor and the working status of the sensor. The data obtained by the sensor is converted into a register and then transferred to the ESP8266 module through serial communication. The data is sent to the cloud by the ESP8266.

\subsection{Network communication module design}

ESP8266 has a complete and self-contained Wi-Fi network function, which can be used independently or run as a slave on other host MCUs. When ESP8266 is used independently, it can be started directly from the external flash. The built-in high-speed buffer memory helps to improve system performance and optimize the storage system. In addition, ESP8266 can be used as a Wi-Fi adapter through SPI/SDIO interface or UART interface, and can be applied to any microcontroller-based design. And ESP8266 integrates antenna switch, radio frequency, power amplifier, low noise amplifier, filter and power management module to meet system design requirements. 


\section{Software design}

\subsection{Electronic control part}

Make full use of STM32F4's rich on-board resources, use the built-in timer of the singlechip microcomputer to accurately delay, and use multiple external interrupts and timer interrupts to execute data acquisition and motion control programs to avoid unnecessary delays in the main program. Use ADC and DAC chips to convert digital signals and analog signals to the external environment. Perform average filtering on the data collected by each sensor to reduce errors. Considering the influence of noise, Kalman filter is used to deal with noise interference.

\section{2 loT part}

Use Baidu's intelligent IoT cloud platform to connect devices and clouds through object access and object shadows, communicate through mqtt protocol, and use Baidu Object Visual to develop visual graphical interfaces to display environmental data in real time, so that users or maintenance personnel can remotely anytime and anywhere Monitor indoor environmental indicators and discover potential hazards in time. Use AiThinkerIDE development environment to configure esp8266 IoT hardware, and use mqttfx to configure protocol layer.

\begin{tabular}{|c|c|c|}
\hline DataTableAPI & \multicolumn{2}{c|}{ PlayerAPI } \\
\hline Tsdblivequery \\
\hline Dmsub \\
\hline Staticcsv \\
\hline Staticjson \\
\hline Simulation \\
\cline { 1 - 2 } \\
\cline { 1 - 2 } & Getdashboardconfig & Unwidgetevent \\
\hline Updatedatatableconfig & Onwidgetdomevent \\
\hline Getwidgetprop & Pausedatatablesourcer \\
\hline Setwidgetprop & Resumedatatablesourcer \\
\hline Onwidgetevent & Isdatatablesourcerpaused \\
\hline
\end{tabular}

Fig. 3. Baidu object visual configuration process.

Connecting to the Baidu cloud platform, through the chip's analog-to-digital conversion function, the power consumption of each electrical appliance is detected, and the server performs data storage and analysis processing to achieve a reasonable distribution of power to each electrical consumer. The display screen displays the ambient temperature, humidity and weather conditions in real time, and the mobile APP realizes light adjustment and temperature adjustment. The visual interface of the IoT family intelligent robot is shown in Figure 4.

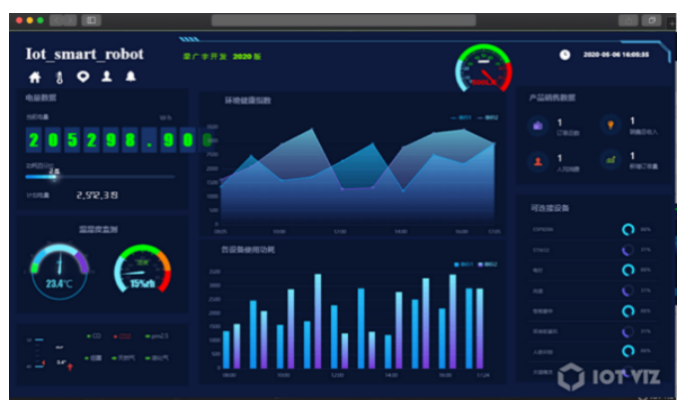

Fig. 4. Visual interface. 


\section{Hardware design}

The omnidirectional movement method is based on a center wheel with many axles located around the wheel, as shown in Figure 5.

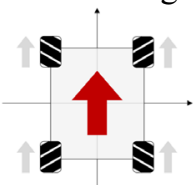

Straight Ahead

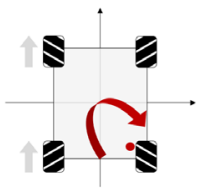

Concerning

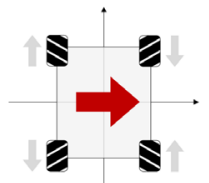

Side Way

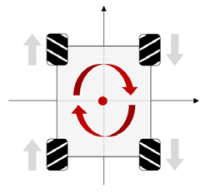

Turn Round

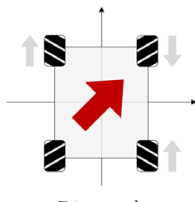

Diagonal

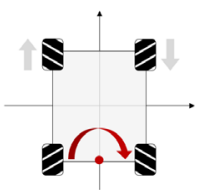

Turn Of Rear Axis

Fig. 5. The influence of the direction of rotation on the direction of the trolley.

In order to control the speed of each wheel, it can realize all-round motions such as forward, lateral, oblique, and rotating [10], as shown in Fig. 8, 9 adopts positional PID control algorithm and incremental PID control algorithm to build a smart space based The interactive multi-resolution display system of the robot system [4], through the intelligent space recognition and calculation of the finger direction to find the area of interest, and control the robot on-board projector to project high-resolution image information. It has fast processing speed and operability. Good advantages.

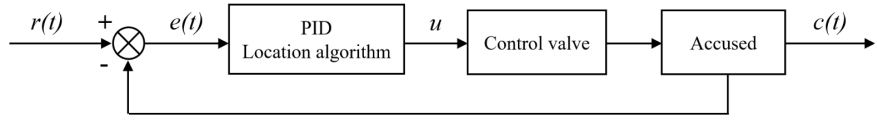

Fig. 8. Position PID control algorithm.

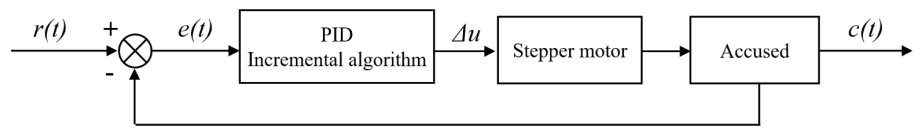

Fig. 9. Incremental PID control algorithm.

\section{Test}

(1) Test ESP8266 networking speed: the average time is 3.2s.

(2) To test the time required for the DC geared motor to perform work at different voltages, it takes $20 \mathrm{~s}$ for a $6 \mathrm{~V}$ single pass, $13.8 \mathrm{~s}$ for a $12 \mathrm{~V}$ single pass, and $7.5 \mathrm{~s}$ for a $18 \mathrm{~V}$ single pass.

(3) Test the release speed of mqtt: the average time is $0.08 \mathrm{~s}$

(4) Test mqtt subscription speed: average time-consuming $0.05 \mathrm{~s}$

(5) Test $\mathrm{ADC}$ acquisition waveform, error $0.4 \% \sim 1 \%$ 


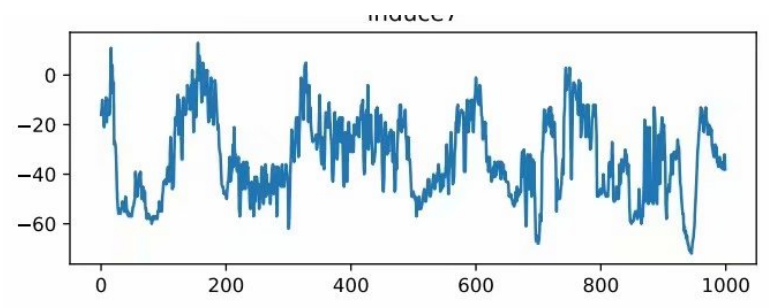

Fig. 10. ADC waveform.

\section{Concluding remarks}

Compared with traditional home intelligent robots, the use of IoT home robots will better serve daily home life. The powerful Internet can store data, and the database can be used to analyze the data, so that it can be more accurately sent to the cloud. The equipment monitors all kinds of household environmental data more accurately, greatly reducing the possibility of fire, gas poisoning, etc. However, the accuracy of indoor GPS positioning has not been solved well, and further exploration and research are needed.

This paper is supported by Tianjin College Student Innovation and Entrepreneurship Training Program(202010066059).

\section{References}

1. Losada Cristina, Mazo Manuel, Palazuelos Sira E,et al.. Identification and tracking of robots in an intelligent space using static cameras and an XPFCP [J]. Robotics and Autonomous Systems, 2013,61 (2):75-85.

2. Lee Jeong-Eom, Park Jiyoung, Kim Gon-Soo, et al.. In-teractive multi-resolution display using a projector mounted mobile robot in intelligent space [J]. Internation-al Journal of Advanced Robotic Systems, 2012,9: 1-7.

3. Morioka Kazuyuki, Oinaga Yudai, Nakamura Yuichi. Control of human-following robot based on cooperative positioning with an intelligentspace [J]. Electronics and Communicationsin Japan, 2012,95 (1): 20-30.

4. Hur Hwan, Ahn Hyo-Sung. Discrete-time $\mathrm{H} \infty$ filtering for mobile robot localization using wirelesssensor network [J]. IEEE Sensors Journal, 2013,13 (1): 245-252.

5. Benjamin Woods. Omni-directional Wheelchair [M]. Centre for Intelligent Information Processing School of Electrical and Electronics Engineering, 2006.

6. N. S. Tlale. Distributed control architecture of an om-ni-directional autonomous guided vehicle [J]. SA Journal of Industrial Engineering, 2005, 16(2): 159-173.

7. Maki K. Habib. Bioinspiration and Robotics [M]. I-Tech, Vienna, Austria, EU, 2007. 